\title{
Correction
}

\section{Correction: Emmons et al., "Rodent Medial Frontal Control of Temporal Processing in the Dorsomedial Striatum"}

In the article "Rodent medial frontal control of temporal processing in the dorsomedial striatum" by Eric B. Emmons, Benjamin J. De Corte, Youngcho Kim, Krystal L. Parker, Matthew S. Matell, and Nandakumar S. Narayanan, which appeared on pages 8718-8733 of the September 6, 2017 issue, the authors discovered a regrettable error in their code, which affected panel I of Figure 11. The correct panel is shown below:

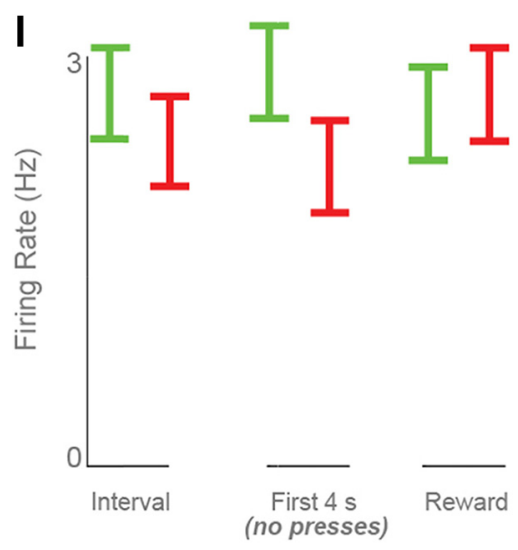

Figure 11.

The legend for Figure 11 should now read:

I, MFC inactivation did not significantly reduce MSN firing around reward events (right).

The following paragraph includes an error and should be ignored in the Results section.

"This effect was apparent in the raw firing rates of MSN neurons during the interval. Indeed, MSN neurons fired significantly less during the interval with MFC inactivated $\left(2.5 \pm 0.1 \mathrm{vs} 1.4 \pm 0.3 \mathrm{~Hz}\right.$ with $\mathrm{MFC}$ inactivated; $t_{(86)}=4.0, p<0.0002$; Fig. $\left.11 I\right)$. This difference held for the first $4 \mathrm{~s}$ of the interval on trials where no presses occurred during this $4 \mathrm{~s}$ epoch $(2.1 \pm 0.2 \mathrm{~Hz}$ with $\mathrm{MFC}$ active vs $1.50 .2 \mathrm{~Hz}$ with MFC inactive; $t_{(86)}=2.4, p<0.02$; Fig. $\left.11 \mathrm{I}\right)$. However, there were no differences for MSN firing during the reward epoch immediately after interval end (12-16 s after cue; $2.1 \pm 0.2 \mathrm{~Hz}$ with MFC active vs $1.7 \pm 0.2 \mathrm{~Hz}$ with MFC inactive; $p=0.19$ ). MFC inactivation did not change the firing rate of DMS MSNs during the entire session $(2.2 \pm 0.3 \mathrm{~Hz}$ with MFC active vs $1.7 \pm 0.2 \mathrm{~Hz}$ with MFC inactive; $p=0.18$ ). These data imply that MFC inactivation decreased the firing of DMS neurons during the interval without changing firing of MSN neurons overall or during the reward epoch.”

Instead, the text in the Results should now read:

"There were no overall differences in firing rate of DMS neurons over the interval with MFC inactivation. (Figure 11I)."

This change does not affect any other figures or results in the manuscript, and does not affect any conclusions or interpretations in the manuscript. The data is now publicly available at: https://narayanan.lab.uiowa.edu/article/datasets.

The authors apologize for any confusion this error caused.

DOI:10.1523/JNEUROSCI.0879-18.2018 